MATHEMATICAL MODELLING OF

POPULATION DYNAMICS

BANACH CENTER PUBLICATIONS, VOLUME 63

INSTITUTE OF MATHEMATICS

POLISH ACADEMY OF SCIENCES

WARSZAWA 2004

\title{
GLOBAL EXISTENCE OF SOLUTIONS FOR A STRONGLY COUPLED POPULATION SYSTEM
}

\author{
GONZALO GALIANO \\ Departamento de Matemáticas, Universidad de Oviedo \\ 33007 Oviedo, Spain \\ E-mail:galiano@correo.uniovi.es
}

ANSGAR JÜNGEL

Fachbereich Mathematik und Statistik, Universität Konstanz

78457 Konstanz, Germany

E-mail: juengel@fmi.uni-konstanz.de

\begin{abstract}
A strongly coupled cross-diffusion model for two competing species in a heterogeneous environment is analyzed. We sketch the proof of an existence result for the evolution problem with non-flux boundary conditions in one space dimension, completing previous results [4]. The proof is based on a symmetrization of the problem via an exponential transformation of variables and the use of an entropy functional.
\end{abstract}

1. Introduction. For the time evolution of two species with non-homogeneous densities, diffusion effects have to be taken into account. Shigesada et al. proposed in their pioneering work [12] to introduce so-called cross-diffusion terms modeling the population pressure due to the mutual interferences among the individuals of the species. Denoting by $n_{i}$ the population density of the $i$-th species $(i=1,2)$, the time-dependent equations can be written in one space dimension as [12]

$$
\begin{aligned}
& \partial_{t} n_{1}-\left(\left(\left(c_{1}+\alpha_{11} n_{1}+\alpha_{12} n_{2}\right) n_{1}\right)_{x}+d_{1} n_{1} U_{x}\right)_{x}=0, \\
& \partial_{t} n_{2}-\left(\left(\left(c_{2}+\alpha_{21} n_{1}+\alpha_{22} n_{2}\right) n_{2}\right)_{x}+d_{2} n_{2} U_{x}\right)_{x}=0,
\end{aligned}
$$

2000 Mathematics Subject Classification: Primary 35K55; Secondary 65N40.

The authors acknowledge partial support from the German-Spanish Bilateral Project DAADAcciones Integradas, Project HA2000-0010. The first author was supported by the Spanish D.G.I. Project BFM2000-1324 and the European RNT Project HPRN-CT2002-00274. The second author was supported by the TMR Project ERB-FMBX-CT97-0157, the Gerhard-Hess Program of the Deutsche Forschungsgemeinschaft, grant JU 359/3, and by the AFF Project of the University of Konstanz, grant 4/00 .

The paper is in final form and no version of it will be published elsewhere. 
in the bounded interval $\Omega \subset \mathbb{R}$ with time $t>0$. Here, $U=U(x)$ is the (given) environmental potential, modeling areas where the environmental conditions are more or less favorable $[10,12]$. The diffusion coefficients $c_{i}$ and $\alpha_{i j}$ are non-negative, and $d_{i} \in \mathbb{R}$ $(i, j=1,2)$.

The above system of equations is completed with non-flux boundary conditions and initial conditions:

$$
\begin{aligned}
\left(\left(\left(c_{1}+\alpha_{11} n_{1}+\alpha_{12} n_{2}\right) n_{1}\right)_{x}+d_{1} n_{1} U_{x}\right) \cdot \nu=0, & \\
\left(\left(\left(c_{2}+\alpha_{21} n_{1}+\alpha_{22} n_{2}\right) n_{2}\right)_{x}+d_{2} n_{2} U_{x}\right) \cdot \nu=0, & \text { on } \partial \Omega \times(0, T), \\
n_{i}(\cdot, 0)=n_{0, i} & \text { in } \Omega, \quad i=1,2,
\end{aligned}
$$

where $\nu$ denotes the exterior unit normal to $\partial \Omega$.

Up to now, only partial results are available in the literature concerning the wellposedness of the problem. We summarize some of the available results for the timedependent equations (see [13] for a review) and refer to [8] for the stationary problem. Global existence of solutions and their qualitive behavior for $\alpha_{11}=\alpha_{22}=\alpha_{21}=0$ have been proved in, e.g., $[9,11]$. In this case, Eq. (2) is only weakly coupled. For sufficiently small cross-diffusion parameters $\alpha_{12}>0$ and $\alpha_{21}>0$ (or equivalently, "small" initial data) and vanishing self-diffusion coefficients $\alpha_{11}=\alpha_{22}=0$, Deuring proved the global existence of solutions [2]. For the case $c_{1}=c_{2}$, a global existence result in one space dimension has been obtained by Kim [7]. Furthermore, under the condition

$$
8 \alpha_{11}>\alpha_{12}, \quad 8 \alpha_{22}>\alpha_{21},
$$

Yagi [14] has shown the global existence of solutions in two space dimensions assuming $\alpha_{12}=\alpha_{21}$. A global existence result for weak solutions in any space dimension under condition (6) can be found in [3]. Condition (6) can be easily understood by observing that in this case, the diffusion matrix induces an elliptic operator. If the condition (6) does not hold, there are choices of $c_{i}, \alpha_{i j}, n_{i} \geq 0$ for which the matrix diffusion is not elliptic, and it is therefore unclear if the problem (1)-(5) can be solved for these data. More recently, Ichikawa and Yamada [5] have improved the results of Yagi, replacing condition (6) by

$$
64 \alpha_{11} \alpha_{22}>\alpha_{12} \alpha_{21} \quad \text { or } \quad 64 \alpha_{11} \alpha_{22}=\alpha_{12} \alpha_{21}>0
$$

They use the same techniques as Yagi combined with suitable energy estimates. From the view-point of mathematical biology, conditions like (6) and (7) mean that self-diffusion or diffusion is dominant over cross-diffusion.

In [4] we showed how the existence of solutions of problem (1), (2), (5) with mixed boundary conditions and Lotka-Volterra source terms can be obtained, without assuming conditions like (6) or (7). In the present paper, we perform several changes in the method of proof to tackle the case of non-flux boundary conditions, as originally proposed in [12]. For simplicity, we considered zero source terms, although more general situations may be treated.

More precisely, we are able to show that for any $c_{i}, a_{i}>0$ there exists a weak solution $u_{1}, u_{2}$ to (1)-(5) such that $u_{1}$ and $u_{2}$ are non-negative. We stress the fact that the nonnegativity property is obtained without the use of the maximum principle. The idea 
of the proof is as follows: The system (1)-(2) is first symmetrized via an exponential transformation of variables. A priori estimates are derived for a perturbed semi-discrete problem by using an entropy functional, yielding $H^{1}$ bounds which are independent of the solutions. The non-negativity property is obtained from the embedding $H^{1}(\Omega) \hookrightarrow L^{\infty}(\Omega)$, which holds only in one space dimension.

Before we state the results and sketch the method of proof, we perform (for a smoother presentation) the following change of unknowns:

$$
u_{1}=\alpha_{21} n_{1}, \quad u_{2}=\alpha_{12} n_{2}, \quad \text { and } \quad q=-U_{x} .
$$

We assume that $\alpha_{12}>0$ and $\alpha_{21}>0$ which is no restriction since if $\alpha_{12}=0$ or $\alpha_{21}=0$, at least one of the equations (1), (2) is weakly coupled, and the results of [11] apply. Eqs. (1)-(5) can be reformulated as

$$
\begin{aligned}
& \partial_{t} u_{i}-\left(c_{i} u_{i x}+2 a_{i} u_{i} u_{i x}+\left(u_{1} u_{2}\right)_{x}+d_{i} u_{i} q\right)_{x}=0 \quad \text { in } \Omega \times(0, T)=: Q_{T}, \\
& \left(c_{i} u_{i x}+2 a_{i} u_{i} u_{i x}+\left(u_{1} u_{2}\right)_{x}+d_{i} u_{i} q\right) \cdot \nu=0 \quad \text { on } \partial \Omega \times(0, T)=: \Gamma_{T}, \\
& u(\cdot, 0)=u_{i}^{0} \quad \text { in } \Omega, \quad i=1,2,
\end{aligned}
$$

where $T>0, u_{1}^{0}=\alpha_{21} n_{0,1}, u_{2}^{0}=\alpha_{12} n_{0,2}$ and $a_{1}=\alpha_{11} / \alpha_{21}, a_{2}=\alpha_{22} / \alpha_{12}$.

2. Existence of solutions of problem (8)-(10). The main result is the following theorem.

TheOREM 1. Assume that $q \in L^{2}\left(Q_{T}\right), u_{i}^{0} \in L^{\infty}(\Omega)$ satisfies $u_{i}^{0} \geq \gamma>0$ in $\Omega$ and $a_{i}, c_{i}>0, d_{i} \in \mathbb{R}, i=1,2$. Then, there exists a weak solution $\left(u_{1}, u_{2}\right)$ of (8)-(10) satisfying $u_{1}, u_{2} \in L^{2}\left(0, T ; H^{1}(\Omega)\right) \cap W^{1,1}\left(0, T ; H^{1}(\Omega)^{*}\right)$ and

$$
u_{1}(x, t), u_{2}(x, t) \geq 0 \quad \text { for }(x, t) \in Q_{T} .
$$

This result can be extended in several ways. First, under suitable restrictions we may include non-trivial source terms in the formulation of (8)-(10), see [4]. In particular, Lotka-Volterra type source terms may be considered. Second, by an appropriate and standard approximation technique we may allow the initial data to be just non-negative. With a more subtle approximation of the problem, we may prove the above result for non-negative self-diffusion coefficients, $c_{i}$.

Proof. The proof consists of several steps.

STEP 1. We work with unknowns which symmetrize the elliptic operator. Introduce $w=\left(w_{1}, w_{2}\right)$ by defining $u_{1}=e^{w_{1}}, u_{2}=e^{w_{2}}$ and set $b(w)=\left(b_{1}(w), b_{2}(w)\right)=\left(e^{w_{1}}, e^{w_{2}}\right)$. With the diffusion coefficients

$$
a_{i i}(w)=c_{i} e^{w_{i}}+2 a_{i} e^{2 w_{i}}+e^{w_{1}+w_{2}}, \quad i=1,2, \quad a_{12}(w)=a_{21}(w)=e^{w_{1}+w_{2}},
$$

Eqs. (8)-(10) are formally equivalent to

$$
\begin{aligned}
\partial_{t} b_{i}(w)- & \left(\sum_{j=1}^{2} a_{i j}(w) w_{j x}+d_{i} b_{i}(w) q\right)_{x}=0, \text { in } Q_{T}, \\
\left(\sum_{j=1}^{2} a_{i j}(w) w_{j x}+d_{i} b_{i}(w) q\right) \cdot \nu & =0 \text { on } \Gamma_{T}
\end{aligned}
$$




$$
w(0)=w^{0} \quad \text { in } \Omega
$$

where $w_{i}^{0}=\log \left(u_{i}^{0}\right), i=1,2$.

STEP 2. In order to solve the above problem, we introduce a perturbed semi-discrete problem. Let $\varepsilon>0, N \in \mathbb{N}$ and $\tau=T / N$, the time step. Given $w^{k-1}$, approximating $w$ in the interval $((k-1) \tau, k \tau]$, we are seeking solutions $w^{k}$ of the elliptic problem

$$
\begin{array}{r}
\frac{b_{i}\left(w^{k}\right)-b_{i}\left(w^{k-1}\right)}{\tau}-\left(\sum_{j=1}^{2} a_{i j}\left(w^{k}\right) w_{j x}^{k}+d_{i} b_{i}\left(w^{k}\right) q^{k}\right)_{x}+\varepsilon\left(b_{i}\left(w^{k}\right)-1\right)=0 \quad \text { in } \Omega \\
\left(\sum_{j=1}^{2} a_{i j}\left(w^{k}\right) w_{j x}^{k}+d_{i} b_{i}\left(w^{k}\right) q^{k}\right) \cdot \nu=0 \quad \text { on } \partial \Omega,
\end{array}
$$

for $k=1, \ldots, N$. Here we defined $q^{k}:=\frac{1}{\tau} \int_{(k-1) \tau}^{k \tau} q(\cdot, t) d t$.

We now introduce the discrete entropy, for $k=0, \ldots, N, \alpha=2 \min \left\{c_{1}, c_{2}\right\}$ :

$$
\eta^{k}=\sum_{i=1}^{2} \int_{\Omega}\left(b_{i}\left(w^{k}\right)\left(w_{i}^{k}-1\right)+1\right)+\alpha \int_{\Omega}\left(b_{i}\left(w^{k}\right)-1-w_{i}^{k}\right) \geq 0 .
$$

We can prove the following entropy-type estimate, which holds in any space dimension.

Lemma 1. Let $w^{k} \in H^{1}\left(\Omega ; \mathbb{R}^{2}\right)$ be a weak solution of (14)-(15). Then there exists a constant $C>0$ independent of $\tau$ and $\varepsilon$ such that for any $k=1, \ldots, N$ and any $\tau, \varepsilon>0$,

$$
\begin{aligned}
& \eta^{k}+\tau \sum_{i=1}^{2} \int_{\Omega}\left(\frac{\alpha^{2}}{4}\left|w_{i x}^{k}\right|^{2}+\alpha\left|\left(e^{w_{i}^{k} / 2}\right)_{x}\right|^{2}+a_{i}\left|\left(e^{w_{i}^{k}}\right)_{x}\right|^{2}+\varepsilon\left|w_{i}^{k}\right|^{2}\right) \leq \eta^{k-1}+C \tau \\
& \int_{\Omega} b_{i}\left(w^{k}\right) \leq C .
\end{aligned}
$$

Proof. The key of the proof of (16) is to use $w_{i}^{k}+\alpha\left(1-b_{i}\left(-w^{k}\right)\right) \in H^{1}(\Omega)$ as a test function in the weak formulation of (14)-(15). Adding the corresponding equations for $i=1$ and $i=2$ gives

$$
\begin{aligned}
& \frac{1}{\tau} \sum_{i=1}^{2} \int_{\Omega}\left(b_{i}\left(w^{k}\right)-b_{i}\left(w^{k-1}\right)\right)\left[w_{i}^{k}+\alpha\left(1-b_{i}\left(-w^{k}\right)\right)\right] \\
& \quad+\sum_{i, j=1}^{2} \int_{\Omega} a_{i j}\left(w^{k}\right) w_{j x}^{k}\left[w_{i}^{k}+\alpha\left(1-b_{i}\left(-w^{k}\right)\right)\right]_{x} \\
& \quad+\varepsilon \sum_{i=1}^{2} \int_{\Omega}\left(b_{i}\left(w^{k}\right)-1\right)\left[w_{i}^{k}+\alpha\left(1-b_{i}\left(-w^{k}\right)\right)\right] \\
& =-\sum_{i=1}^{2} \int_{\Omega} d_{i} b_{i}\left(w^{k}\right) q^{k} \cdot\left[w_{i}^{k}+\alpha\left(1-b_{i}\left(-w^{k}\right)\right)\right]_{x}
\end{aligned}
$$

In order to estimate the first term on the left-hand side of (18), we use the convexity of $s \mapsto b_{i}(s)$ and the elementary inequality $e^{s} \geq 1+s$ for all $s \in \mathbb{R}$ :

$$
\frac{1}{\tau} \sum_{i=1}^{2} \int_{\Omega}\left(b_{i}\left(w^{k}\right)-b_{i}\left(w^{k-1}\right)\right)\left[w_{i}^{k}+\alpha\left(1-b_{i}\left(-w^{k}\right)\right)\right]
$$




$$
\begin{aligned}
= & \frac{1}{\tau}\left(\eta^{k}-\eta^{k-1}\right)+\frac{1}{\tau} \sum_{i=1}^{2} \int_{\Omega}\left(b_{i}\left(w^{k}\right)-b_{i}\left(w^{k-1}\right)-b_{i}\left(w^{k-1}\right)\left(w_{i}^{k}-w_{i}^{k-1}\right)\right) \\
& +\frac{\alpha}{\tau} \sum_{i=1}^{2} \int_{\Omega}\left(b_{i}\left(w^{k-1}\right) b_{i}\left(-w^{k}\right)+w_{i}^{k}-w_{i}^{k-1}-1\right) \geq \frac{1}{\tau}\left(\eta^{k}-\eta^{k-1}\right) .
\end{aligned}
$$

We rewrite the second term on the left-hand side of (18) as

$$
\begin{aligned}
& \sum_{i, j=1}^{2} \int_{\Omega} a_{i j}\left(w^{k}\right) w_{j x}^{k}\left(w_{i}^{k x}+\alpha b_{i}\left(-w^{k}\right) w_{i x}^{k}\right)_{x} \\
& =\sum_{i=1}^{2} \int_{\Omega}\left(c_{i} e^{w_{i}^{k}}+2 a_{i} e^{2 w_{i}^{k}}+\alpha c_{i}+2 \alpha a_{i} e^{w_{i}^{k}}\right)\left|w_{i x}^{k}\right|^{2} \\
& \quad+\alpha \int_{\Omega}\left(e^{w_{2}^{k}}\left|w_{1 x}^{k}\right|^{2}+e^{w_{1}^{k}}\left|w_{2 x}^{k}\right|^{2}+\left(e^{w_{1}^{k}}+e^{w_{2}^{k}}\right) w_{1 x}^{k} w_{2 x}^{k}\right)+4 \int_{\Omega}\left|\left(e^{\left(w_{1}^{k}+w_{2}^{k}\right) / 2}\right)_{x}\right|^{2} .
\end{aligned}
$$

For the third term on the left-hand side, it is easy to check that $\left(e^{s}-1\right)\left(s+\alpha\left(1-e^{-s}\right)\right) \geq s^{2}$. Finally, for the first term on the right-hand side of (18) we employ Young's inequality:

$$
\begin{aligned}
& -\sum_{i=1}^{2} \int_{\Omega} d_{i} b_{i}\left(w^{k}\right) q^{k}\left(\nabla w_{i}^{k}+\alpha e^{-w_{i}^{k}} w_{i x}^{k}\right) \\
& \leq \sum_{i=1}^{2} \int_{\Omega}\left(a_{i} e^{2 w_{i}^{k}}\left|w_{i x}^{k}\right|^{2}+\frac{\alpha c_{i}}{2}\left|w_{i x}^{k}\right|^{2}+\left(\frac{1}{4}+\frac{1}{2 \alpha c_{i}}\right) d_{i}^{2}\left|q_{k}\right|^{2}\right) .
\end{aligned}
$$

Putting the above estimates together, we infer from (18):

$$
\begin{aligned}
& \frac{1}{\tau}\left(\eta^{k}-\eta^{k-1}\right)+\sum_{i=1}^{2} \int_{\Omega}\left(\left(\frac{\alpha c_{i}}{2}+\left(c_{i}+2 \alpha a_{i}\right) e^{w_{i}^{k}}+a_{i} e^{2 w_{i}^{k}}\right)\left|w_{i x}^{k}\right|^{2}+\varepsilon\left|w_{i}^{k}\right|^{2}\right) \\
& \leq \sum_{i=1}^{2} \int_{\Omega}\left(\frac{1}{4}+\frac{1}{2 \alpha c_{i}}\right) d_{i}^{2}\left|q_{k}\right|^{2}-\alpha \int_{\Omega}\left(e^{w_{2}^{k}}\left|w_{1 x}^{k}\right|^{2}+e^{w_{1}^{k}}\left|w_{2 x}^{k}\right|^{2}+\left(e^{w_{1}^{k}}+e^{w_{2}^{k}}\right) w_{1 x}^{k} \cdot w_{2 x}^{k}\right) \\
& \leq C+\frac{\alpha}{4} \int_{\Omega}\left(e^{w_{1}^{k}}\left|w_{1 x}^{k}\right|^{2}+e^{w_{2}^{k}}\left|w_{2 x}^{k}\right|^{2}\right) .
\end{aligned}
$$

The last integral can be absorbed by the second term on the left-hand side since $\alpha=$ $2 \min \left\{c_{1}, c_{2}\right\}$ and (16) follows.

To prove (17) we use $\psi=1$ as a test function in the weak formulation of (14)-(15). We obtain, for $k=1, \ldots, N$,

$$
\int_{\Omega} e^{w_{i}^{k}}=\int_{\Omega} e^{w_{i}^{k-1}}-\varepsilon \tau \int_{\Omega}\left(e^{w_{i}^{k}}-1\right) \leq \int_{\Omega} e^{w_{i}^{k-1}}+\varepsilon \tau|\Omega| .
$$

Therefore, we obtain

$$
\int_{\Omega} e^{w_{i}^{k}} \leq \int_{\Omega} e^{w_{i}^{0}}+\varepsilon k \tau|\Omega|,
$$

and since $k \tau \leq T$ and $\varepsilon>0$ is small (say $\varepsilon<1$ ), estimate (17) follows.

STEP 3. We consider the linear problem

$$
\frac{b_{i}(z)-b_{i}\left(w^{k-1}\right)}{\tau}-\left(\sum_{j=1}^{2} a_{i j}(z) w_{j x}^{k}+d_{i} b_{i}(z) q^{k}\right)_{x}+\varepsilon \frac{b_{i}(z)-1}{z_{i}} w_{i}^{k}=0 \quad \text { in } \Omega,
$$




$$
\left(\sum_{j=1}^{2} a_{i j}(z) w_{j x}^{k}+d_{i} b_{i}(z) q^{k}\right) \cdot \nu=0 \quad \text { on } \partial \Omega,
$$

for $w^{k-1}, z \in H^{1}(\Omega)$. By the Lax-Milgram lemma, this problem admits a unique solution $w \in H^{1}(\Omega)$. Note that since the spatial dimension is one, the $H^{1}(\Omega)$ estimate for $w$ provides an $L^{\infty}(\Omega)$ estimate of $w$. We may then use the Leray-Schauder fixed-point theorem to prove the existence of weak solutions of the semi-discrete problem (14)-(15).

LEMma 2. Let $w^{k-1} \in L^{\infty}\left(\Omega ; \mathbb{R}^{2}\right), k \geq 1$. Then there exists a weak solution $w^{k} \in$ $H^{1}\left(\Omega ; \mathbb{R}^{2}\right)$ of (14)-(15).

The proof relies in the compact embedding $L^{\infty}(\Omega) \subset H^{1}(\Omega)$. For a similar result, see Lemma 3 of [4].

STEP 4. We define the piecewise constant functions $w^{(\tau)}$ by

$$
w^{(\tau)}(x, t)=w^{k}(x) \quad \text { if }(x, t) \in \Omega \times((k-1) \tau, k \tau]
$$

and $q^{(\tau)}$ in a similar way. It is clear that

$$
q^{(\tau)} \rightarrow q \quad \text { in } L^{2}\left(Q_{T}\right) \text { as } \tau \rightarrow 0 .
$$

The following result is a direct consequence of Lemma 1 and Poincaré's inequality in the form $\|z\|_{H^{1}(\Omega)} \leq C\left(\left\|z_{x}\right\|_{L^{2}(\Omega)}+\left|\int_{\Omega} z\right|\right)$.

COROLlary 1. Let $\tau>0$. Then the following estimates hold:

$$
\begin{aligned}
& \left\|\eta^{(\tau)}\right\|_{L^{\infty}\left(0, T ; L^{1}(\Omega)\right)} \leq C, \\
& \| b_{i}\left(w^{(\tau)} \|_{L^{\infty}\left(0, T ; L^{1}(\Omega)\right)} \leq C,\right. \\
& \sum_{i=1}^{2}\left(\alpha\left\|e^{w_{i}^{(\tau)} / 2}\right\|_{L^{2}\left(0, T ; H^{1}(\Omega)\right)}^{2}+a_{i}\left\|e^{w_{i}^{(\tau)}}\right\|_{L^{2}\left(0, T ; H^{1}(\Omega)\right)}^{2}+\varepsilon\left\|w_{i}^{(\tau)}\right\|_{L^{2}(0, T ; \Omega)}^{2}\right) \leq C,
\end{aligned}
$$

where $C>0$ is independent of $\tau$ and $\varepsilon$, and

$$
\eta^{(\tau)}(t)=\sum_{i=1}^{2} \int_{\Omega}\left(b_{i}\left(w^{(\tau)}\right)\left(w_{i}^{(\tau)}-1\right)+1+\alpha\left(b_{i}\left(w^{(\tau)}\right)-1-w_{i}^{(\tau)}\right)\right)(t) .
$$

To pass to the limit, we also need estimates for the discrete time derivative. For this we define

$$
\tilde{b}^{(\tau)}(\cdot, t)=\frac{k \tau-t}{\tau}\left(b\left(w^{k}\right)-b\left(w^{k-1}\right)\right)+b\left(w^{k}\right), \quad t>0,
$$

and introduce $\sigma_{\tau}$, the shift operator:

$$
\sigma_{\tau} w^{(\tau)}(\cdot, t)=w^{k-1} \quad \text { if } t \in((k-1) \tau, k \tau], \quad k=1, \ldots, N .
$$

LEMMA 3. We have

$\tau^{-1}\left\|b\left(w^{(\tau)}\right)-b\left(\sigma_{\tau} w^{(\tau)}\right)\right\|_{L^{1}\left(0, T ; H^{1}(\Omega)^{*}\right)}+\left\|\partial_{t} \tilde{b}^{(\tau)}\right\|_{L^{1}\left(0, T ; H^{1}(\Omega)^{*}\right)}+\left\|\tilde{b}^{(\tau)}\right\|_{L^{2}\left(0, T ; H^{1}(\Omega)\right)} \leq C$, where $C$ does not depend on $\tau, \varepsilon$. 
Proof. From the weak formulation of Eqs. (14) for $i=1,2$ we obtain for $i=1,2$,

$$
\begin{aligned}
& \tau^{-1}\left\|b_{i}\left(w^{(\tau)}\right)-b_{i}\left(\sigma_{\tau} w^{(\tau)}\right)\right\|_{L^{1}\left(0, T ; H^{1}(\Omega)^{*}\right)} \\
& \leq\left\|c_{i}\left(e^{w_{i}^{(\tau)}}\right)_{x}+2 a_{i} e^{w_{i}^{(\tau)}}\left(e^{w_{i}^{(\tau)}}\right)_{x}+d_{i} e^{w_{i}^{(\tau)}} q^{(\tau)}+\varepsilon\left(b_{i}\left(w^{(\tau)}\right)-1\right)\right\|_{L^{1}\left(0, T ; L^{2}(\Omega)\right)} \\
& \quad+\left\|e^{w_{1}^{(\tau)}}\left(e^{w_{2}^{(\tau)}}\right)_{x}+e^{w_{2}^{(\tau)}}\left(e^{w_{1}^{(\tau)}}\right)_{x}\right\|_{L^{1}\left(0, T ; L^{2}(\Omega)\right)} \\
& \leq c_{i}\left\|\left(e^{w_{i}^{(\tau)}}\right)_{x}\right\|_{L^{1}\left(0, T ; L^{2}(\Omega)\right)}+2 a_{i}\left\|e^{w_{i}^{(\tau)}}\right\|_{L^{2}\left(0, T ; L^{\infty}(\Omega)\right)}\left\|\left(e^{w_{i}^{(\tau)}}\right)_{x}\right\|_{L^{2}\left(Q_{T}\right)} \\
& \quad+d_{i}\left\|e^{w_{i}^{(\tau)}}\right\|_{L^{2}\left(0, T ; L^{\infty}(\Omega)\right)}\left\|q^{(\tau)}\right\|_{L^{2}\left(Q_{T}\right)}+\varepsilon\left(\left\|e^{w_{i}^{(\tau)}}\right\|_{L^{1}\left(0, T ; L^{2}(\Omega)\right)}+T|\Omega|^{2}\right) \\
& \left.\quad+\left\|e^{w_{1}^{(\tau)}}\right\|_{L^{2}\left(0, T ; L^{\infty}(\Omega)\right)} \| / e^{w_{2}^{(\tau)}}\right)_{x}\left\|_{L^{2}\left(Q_{T}\right)}+\right\| e^{w_{2}^{(\tau)}}\left\|_{L^{2}\left(0, T ; L^{\infty}(\Omega)\right)}\right\|\left(e^{w_{1}^{(\tau)}}\right)_{x} \|_{L^{2}\left(Q_{T}\right)} .
\end{aligned}
$$

Using Poincaré's inequality and that spatial dimension is one, we obtain

$$
\left\|e^{w_{i}^{(\tau)}}\right\|_{L^{2}\left(0, T ; L^{\infty}(\Omega)\right)} \leq C\left\|e^{w_{i}^{(\tau)}}\right\|_{L^{2}\left(0, T ; H^{1}(\Omega)\right)} \leq C,
$$

in view of Corollary 1 , and therefore

$$
\left\|b_{i}\left(w^{(\tau)}\right)-b_{i}\left(\sigma_{\tau} w^{(\tau)}\right)\right\|_{L^{1}\left(0, T ; H^{1}(\Omega) *\right.} \leq C \tau .
$$

Furthermore,

$$
\left\|\partial_{t} \tilde{b}^{(\tau)}\right\|_{L^{1}\left(0, T, H^{1}(\Omega)^{*}\right)} \leq \tau^{-1}\left\|b\left(w^{(\tau)}\right)-b\left(\sigma_{\tau} w^{(\tau)}\right)\right\|_{L^{1}\left(0, T ; H^{1}(\Omega)^{*}\right)} \leq C,
$$

and

$$
\left\|\tilde{b}^{(\tau)}\right\|_{L^{2}\left(0, T ; H^{1}(\Omega)\right)} \leq 2\left\|b\left(w^{(\tau)}\right)\right\|_{L^{2}\left(0, T ; H^{1}(\Omega)\right)}+\left\|b\left(\sigma_{\tau} w^{(\tau)}\right)\right\|_{L^{2}\left(0, T ; H^{1}(\Omega)\right)} \leq C,
$$

by Corollary 1.

STEP 5. We already have all the necessary estimates to pass to the limit $\tau \rightarrow 0$ and $\varepsilon \rightarrow 0$. In fact, we take $\varepsilon=\tau$ and perform the limit $\varepsilon=\tau \rightarrow 0$. Since the embedding $H^{1}(\Omega) \hookrightarrow L^{\infty}(\Omega)$ is compact in one space dimension, we can apply Aubin's lemma to $\tilde{b}^{(\tau)}$, in view of the uniform bounds of Lemma 3 , to obtain, up to a subsequence which is not relabeled,

$$
\begin{gathered}
\partial_{t} \tilde{b}^{(\tau)} \rightarrow \partial_{t} z \text { weakly in } L^{1}\left(0, T ; H^{1}(\Omega)^{*}\right), \\
\tilde{b}^{(\tau)} \rightarrow z \text { weakly in } L^{2}\left(0, T ; H^{1}(\Omega)\right), \\
\tilde{b}^{(\tau)} \rightarrow z \text { strongly in } L^{2}\left(0, T ; L^{\infty}(\Omega)\right), \\
b\left(w^{(\tau)}\right) \rightarrow u \text { weakly in } L^{2}\left(0, T ; H^{1}(\Omega)\right) .
\end{gathered}
$$

By Lemma 3 we have, as $\tau \rightarrow 0$,

$$
\left\|\tilde{b}^{(\tau)}-b\left(w^{(\tau)}\right)\right\|_{L^{1}\left(0, T ; H^{1}(\Omega)^{*}\right)} \leq\left\|b\left(w^{(\tau)}\right)-b\left(\sigma_{\tau} w^{(\tau)}\right)\right\|_{L^{1}\left(0, T ; H^{1}(\Omega)^{*}\right)} \rightarrow 0,
$$

and hence $z=u$. Finally, using the above estimates and convergences, we are able to prove that

$$
e^{w_{i}^{(\tau)}}=b_{i}\left(w^{(\tau)}\right) \rightarrow u_{i} \quad \text { strongly in } L^{2}\left(Q_{T}\right), \quad i=1,2,
$$

(see [4], for a similar result).

Now we can let $\tau=\varepsilon \rightarrow 0$ in the weak formulation of (14), $i=1,2$, which reads for $\phi \in L^{\infty}\left(0, T ;\left(W^{1, \infty}(\Omega)\right)^{*}\right)$ : 


$$
\begin{gathered}
\int_{0}^{T}\left\langle\partial_{t}{\tilde{b_{i}}}^{(\tau)}, \phi\right\rangle+\int_{Q_{T}}\left(c_{i}\left(e^{w_{i}^{(\tau)}}\right)_{x}+2 a_{i} e^{w_{i}^{(\tau)}}\left(e^{w_{i}^{(\tau)}}\right)_{x}+\left(e^{w_{1}^{(\tau)}+w_{2}^{(\tau)}}\right)_{x}\right) \phi_{x} \\
=-\varepsilon \int_{Q_{T}}\left(e^{w_{i}^{(\tau)}}-1\right) \phi-d_{i} \int_{Q_{T}} e^{w_{i}^{(\tau)}} q^{(\tau)} \phi_{x} .
\end{gathered}
$$

In view of (22)-(26) and (21) we obtain

$$
\int_{0}^{T}\left\langle\partial_{t} u_{i}, \phi\right\rangle+\int_{Q_{T}}\left(c_{i} u_{i x}+2 a_{i} u_{i} u_{i x}+\left(u_{1} u_{2}\right)_{x}\right) \phi_{x}=-d_{i} \int_{Q_{T}} u_{i} q \phi_{x},
$$

i.e. $u=\left(u_{1}, u_{2}\right)$ is a weak solution of (8)-(9). Moreover, the initial condition (10) is satisfied in the sense of $H^{1}(\Omega)^{*}$.

\section{References}

[1] P. Degond, S. Génieys, and A. Jüngel, Symmetrization and entropy inequality for general diffusion equations, C. R. Acad. Sci. Paris 325 (1997), 963-968.

[2] P. Deuring, An initial-boundary value problem for a certain density-dependent diffusion system, Math. Z. 194 (1987), 375-396.

[3] G. Galiano, M. Garzón, and A. Jüngel, Analysis and numerical solution of a nonlinear cross-diffusion system arising in population dynamics, Rev. Real Academia de Ciencias Serie A. Mat. 95 (2001), 281-295.

[4] G. Galiano, M. Garzón, and A. Jüngel, Semi-discretization in time and numerical convergence of solutions of a nonlinear cross-diffusion system arising in population dynamics, Numer. Math., to appear.

[5] T. Ichikawa and Y. Yamada, Some remarks on global solutions to some quasilinear parabolic system with cross-diffusion, Funkcialaj Ekvacioj 43 (2000), 285-301.

[6] A. Jüngel, Quasi-hydrodynamic Semiconductor Equations, Progr. Nonlinear Differential Equations Appl., Birkhäuser, Basel, 2001.

[7] J. U. Kim, Smooth solutions to a quasi-linear system of diffusion equations for a certain population model, Nonlin. Anal. 8 (1984), 1121-1144.

[8] Y. Lou and W.-M. Ni, Diffusion vs. cross-diffusion: an elliptic approach, J. Diff. Eqs. 154 (1999), 157-190.

[9] Y. Lou, W.-M. Ni, and Y. Wu, The global existence of solutions for a cross-diffusion system, Adv. Math. (Beijing) 25 (1996), 283-284.

[10] M. Mimura and K. Kawasaki, Spatial segregation in competitive interaction-diffusion equations, J. Math. Biol. 9 (1980), 49-64.

[11] M. Pozio and A. Tesei, Global existence of solutions for a strongly coupled quasilinear parabolic system, Nonlin. Anal. 14 (1990), 657-689.

[12] N. Shigesada, K. Kawasaki, and E. Teramoto, Spatial segregation of interacting species, J. Theor. Biol. 79 (1979), 83-99.

[13] Y. Wu, Qualitative studies of solutions for some cross-diffusion systems, in: T.-T. Li, M. Mimura, Y. Nishiura, and Q.-X. Ye (eds.), China-Japan Symposium on ReactionDiffusion Equations and Their Applications and Computational Aspects, World Scientific, Singapore, 1997, 177-187.

[14] A. Yagi, Global solution to some quasilinear parabolic system in population dynamics, Nonlin. Anal. 21 (1993), 603-630. 